\title{
The cell biology behind the oncogenic PIP3 lipids
}

\author{
Ana C. Carrera ${ }^{1, *}$ and Richard Anderson ${ }^{2}$
}

\begin{abstract}
The different mechanisms of phosphoinositide 3-kinase (PI3K) activation in cancer as well as the events that result in PI3K pathway reactivation after patient treatment with $\mathrm{PI} 3 \mathrm{~K}$ inhibitors was discussed on October 15-17th, 2018, in the medieval town of Baeza (Universidad Internacional de Andalucía, Spain) at the workshop entitled 'The cell biology behind the oncogenic PIP3 lipids'. These topics and the data presented regarding cellular functions altered by PI3K deregulation, the cooperation of PI3K/PTEN mutations with other tumor drivers, and the lessons learned for PI3K-targeted therapy, are discussed below.
\end{abstract}

\section{INTRODUCTION}

The phosphoinositide 3-kinase (PI3K) family comprises eight kinases divided into three classes. Overactivation of class I PI3Ks (PI3K $\alpha$, $\mathrm{PI} 3 \mathrm{~K} \beta, \mathrm{PI} 3 \mathrm{~K} \gamma$ and $\mathrm{PI} 3 \mathrm{~K} \delta$ ) is a common event in cancer. Indeed, activating mutations in $\mathrm{PI} 3 \mathrm{~K} \alpha$ and genetic alterations of the tumor suppressor PTEN (a lipid phosphatase that opposes class I PI3K action) are the second and third, respectively, more frequent mutations in cancer (Martincorena and Campbell, 2015). Class I enzymes produce phosphatidylinositol 3,4,5-trisphosphate [PI(3,4,5)P3] from phosphatidylinositol 4,5-bisphosphate [PI(4,5)P2], class III PI3Ks produce phosphatidylinositol 3-phosphate [PI(3)P], and class II enzymes generate PI(3)P and phosphatidylinositol 3,4-bisphosphate $[\mathrm{PI}(3,4) \mathrm{P} 2]$; these three classes have all been linked, in one way or another, to cancer pathology.

Given the frequency of class I PI3K pathway activation in cancer, considerable efforts have been made to test, as quickly as possible, the clinical outcome of PI3K inhibitor-based treatments. Unfortunately, the clinical impact of these therapeutic agents for solid tumors has been somewhat discouraging due to deactivation of negative regulatory mechanisms, acquisition of resistance, low tolerability of the drugs, or simply a lack of effective pathway inhibition in vivo, and has resulted in limited success. For these reasons, it is imperative to develop new strategies based on a deeper understanding of the basic principles behind PI3K action. This workshop provided an opportunity for open discussion with the aim of more clearly assessing the impact of PI3K pathway mutations in human pathology, which should lead to novel strategies for controlling PI3K in cancer.

\section{Mechanisms of PI3K activation in cancer}

The structural studies presented by Roger L. Williams (MRC Laboratory of Molecular Biology, UK) suggest that activation of $\mathrm{PI} 3 \mathrm{~K} \alpha$, mediated either by its $\mathrm{p} 85$ regulatory subunit or by Ras,

1Department of Immunology and Oncology, Centro Nacional de Biotecnología/ CSIC, Darwin 3, Madrid 28049, Spain. 'University of Wisconsin School of Medicine and Public Health, 1300 University Avenue, Madison, WI 53706-1532, USA.

*Author for correspondence (acarrera@cnb.csic.es)

Received 28 November 2018; Accepted 28 November 2018 favors the interaction of PI3K with the plasma membrane. Thus, blocking the plasma membrane recruitment of $\mathrm{PI} 3 \mathrm{~K} \alpha$ could be an effective therapeutic approach.

Activation of PI3K is triggered by ligand binding to receptor tyrosine kinases (RTKs; EGF receptor etc.) and is enhanced by Ras (Jiménez et al., 2002). The work of Jonathan M. Backer (Albert Einstein College of Medicine, NY) showed that, in addition to RTK-mediated activation, agonist binding to $G$ protein-coupled receptors (GPCRs) also activates PI3K $\beta$; simultaneous switch on of RTK and GPCRs induces optimal PI3K $\beta$ activation (Houslay et al., 2016) (Fig. 1). PI3K $\beta$ is not frequently mutated in cancer; however, its overexpression is found in several cancer types and can trigger cell transformation (Liu et al., 2009; Denley et al., 2007). Jonathan's studies on the contribution of PI3K $\beta$ to breast cancer metastasis illustrate that GPCR signaling to PI3K $\beta$ is required for the formation of invadopodia (Khalil et al., 2016). In addition, his group has highlighted that, beyond its role in cancer cells, PI3K $\beta$ also modulates the capacity of macrophages to degrade extracellular matrix. Thus, the diversity in activating signals, as well as the cell type involved, results not only in PI3K-driven cancer cell survival but also increases the capacity of cancer cells to invade.

Approaching these matters from a different angle, Len Stephens and his team (Babraham Institute, UK) have examined why phosphoinositides have a unique profile of acyl chains in their diacylglycerol skeleton (enriched in stearoyl-arachidonoyl species) when compared with closely related phospholipids. They conclude that the acyl chain composition changes in different cell types as tumorigenesis progresses and upon loss of PTEN function, suggesting that changes in acyl chain composition are in fact subject to complex regulation.

Beyond the class I-mediated $\mathrm{PI}(3,4,5) \mathrm{P} 3$ formation at the membrane, Vps34 (class III)-mediated regulation of autophagy is also critical for cancer cell survival (reviewed in Ohashi et al., 2018). The in vitro structural studies by Roger L. Williams have unmasked how Vsp34 being a component of two complexes (complex I or II) can control autophagy or endocytosis.

Mutations in the PI3K class II (PI3K-C2) family proteins are also found in breast cancer tumors (Gulluni et al., 2017). PI3K-C2 generates $\mathrm{PI}(3) \mathrm{P}$ as well as $\mathrm{PI}(3,4) \mathrm{P} 2$, a lipid that is also able to activate the survival pathway mediated by AKT (Franke et al., 1997). PI(3)P produced by PI3K-C2 $\alpha$ inside endomembranes is important for Rab11-mediated endocytic recycling (Campa et al., 2018) and the trafficking of cargo to the primary cilium (Franco et al., 2014). On the other hand, PI3K-C2 $\alpha$-derived PI $(3,4) \mathrm{P} 2$ is important in endocytosis (Posor et al., 2013). The results presented by Dr Emilio Hirsch (University of Torino, Italy) showed that PI3K-C2 $\alpha$ also has a kinase-independent function involved in mitotic completion. Indeed, PI3K-C2 $\alpha$ is a limiting scaffold protein that organizes the clathrin and TACC3 complex, crosslinking kinetochore fibers during mitosis.

Thus, PI3K $\beta$ activation by $\beta 1$-integrin receptors in tumor cells enhances metastasis; in addition, its activation by GPCRs in 


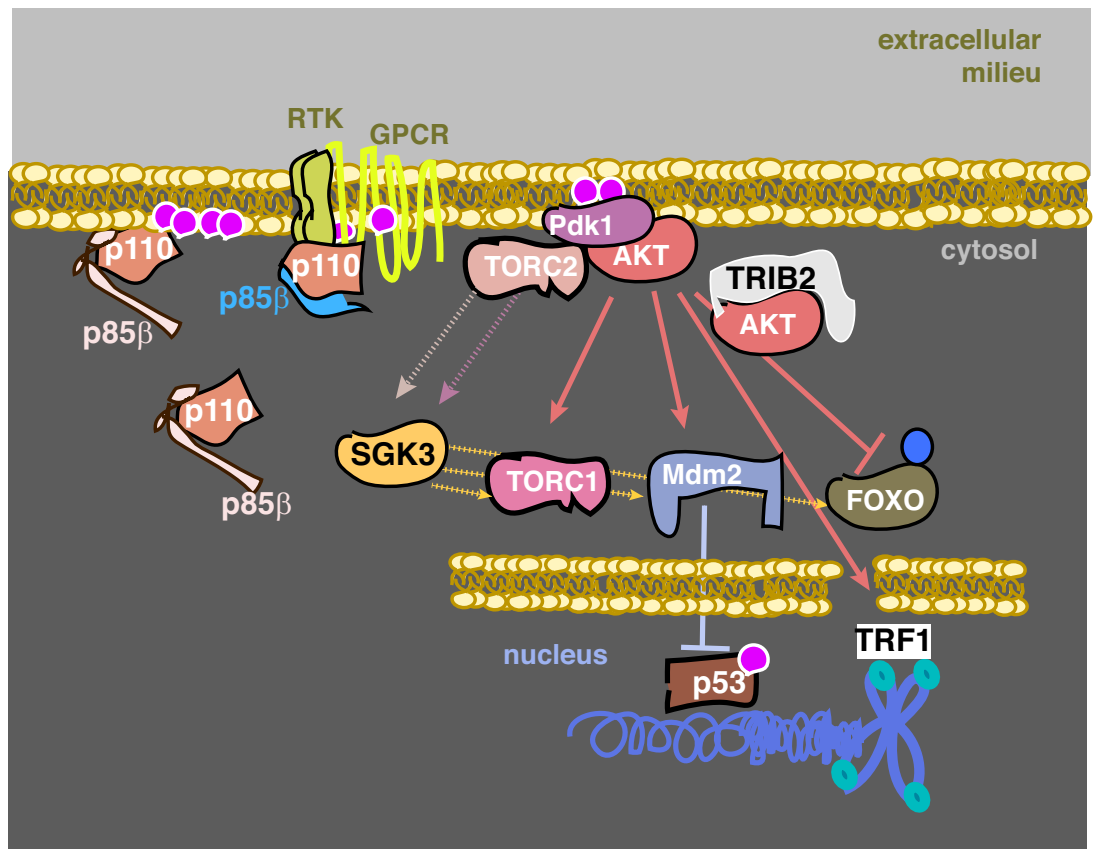

Fig. 1. Novel findings in the oncogenic PI3K/AKT pathway. The figure highlights that, apart from receptor RTK activation, association with GPCRs also affects activation of PI3K (note PI3K is shown in the figure as a complex of its subunits p85 and p110), particularly in the case of PI3K $\beta$. The composition of the p85-p110 complex changes in some tumor types as they progress, with the $p 85 \alpha$ regulatory isoform being frequently downregulated, and/or the p85 $\beta$ being overexpressed; this switch in p85 subunit usage contributes to tumor progression. Activation of AKT involves the action of protein kinase D1 (Pdk1) and the TORC2 complex downstream of PI3K. In addition to the well-known substrates [FOXO transcription factors and TSC2 (a regulator of the TORC1 complex)], TRF1, a component of the shelterin complex, which protects telomere integrity in chromosomes, is also a substrate of AKT; PI3K/AKT inhibition impairs telomere integrity. Another well-known substrate of AKT, Mdm2, is a negative regulator of p53. Nuclear p53 can be stabilized by direct binding to poly-phosphoinositides. Following sustained AKT inhibition (i.e. in cancer treatment), SGK3, a kinase similar to AKT, might acquire the capacity of phosphorylating AKT substrates,

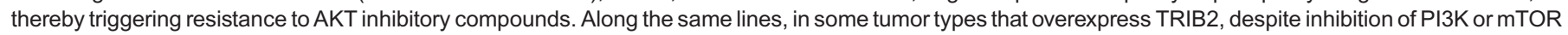
(the core kinase of TORC1 complexes), AKT can remain active owing to the interaction with TRIB2. (For simplicity, a fragment of single lipid bilayer is represented to separate the cytosol and the nucleus).

macrophages modulates invasion of nearby tumor cells. The activation of $\mathrm{PI} 3 \mathrm{~K} \alpha$ is completed at the plasma membrane, which is the location that needs to be targeted to avoid its action in cancer. Besides class I enzymes, the activity of class II and class III enzymes might be considered as targets for the treatment of tumors exhibiting PI3K-C2 $\alpha$ mutations (class II) or tumors dependent on autophagy for survival (class III).

\section{PI3K reactivation after inhibitor treatment in patients}

Three important contributions were presented in the meeting regarding PI3K pathway adaptation to treatment with inhibitory compounds. Athanasios Karapetsas (Dario Alessi's laboratory; MRC Protein Phosphorylation and Ubiquitylation Unit, Dundee, UK) presented one of the reactivation mechanisms of the PI3K/AKT pathway detected in cancer after prolonged treatment with AKT inhibitors. Under these conditions, SGK3, a kinase structurally similar to AKT, acquires the capacity to phosphorylate AKT substrates (Malik et al., 2018), thereby activating the pathway (Fig. 1). Accordingly, targeting SGK3 after prolonged AKT inhibitor treatment might help to deactivate the PI3K/AKT pathway.

Wolfgang Link (Instituto de Investigaciones Biomédicas/CSIC, Spain) described a novel mechanism of resistance to PI3K or mTOR inhibitors in melanoma mediated by the kinase-like protein TRIB2 (Link, 2015). In these tumors, TRIB2 overexpression is able to maintain active AKT through the direct interaction of the TRIB2 Cterminal COP1 binding domain with AKT (Fig. 1). This interaction promotes the phosphorylation of AKT at S473; the active status of AKT is maintained even when upstream regulators, such as PI3K or
mTOR, are inhibited. The TRIB2-mediated activation of AKT leads to the attenuation of FOXO family proteins and p53 activity, as shown in mice and in samples from patients with melanoma (Hill et al., 2017). The fact that TRIB2 confers drug resistance by inactivating these two major effectors of anticancer drug action raises the exciting possibility of using TRIB2 as a biomarker to stratify melanoma patients to specific treatment options. TRIB2 is also highly expressed in several other human malignancies (Hill et al., 2015, 2017), and inhibition of PI3K or mTOR in these tumors might not be useful unless patients are treated to overcome TRIB2mediated resistance.

Rafael Pulido (Ikerbasque, Spain) presented data showing that mutations in PTEN, the negative regulator of the class I PI3K product $\mathrm{PI}(3,4,5) \mathrm{P} 3$, not only affect its activity but might also alter its location. PTEN normally enters and exits the nucleus, but only inactivates the PI3K pathway when it localizes to the plasma membrane. Thus, a mutated PTEN that does not reach the membrane could behave as a loss-of-function protein and favor enhanced PI3K activation. Indeed, the PTEN Q17E mutant concentrates in the nucleus, showing a reduced effect on the plasma membrane $\mathrm{PI}(3,4,5) \mathrm{P} 3$. Interestingly, the same mutation in the PTEN-Long isoform (with an extended N-terminal region) does not lead to its sequestration in the nucleus, but controls, although with less activity, the regulation of $\mathrm{PI}(3,4,5) \mathrm{P} 3$ levels at the membrane.

Overall, when considering the effects of PTEN mutations, we should take into account that the action could be different in the PTEN-Long isoform. In addition, we heard about two novel 
resistance mechanisms: overexpression of TRIB2 and change of substrate specificity by SGK3.

\section{Consequences of sustained PI3K activation for different tissue and cell responses}

The endothelium is highly dependent on PI3K activity. The work of Mariona Graupera (Institut d'Investigació Biomèdica de Bellvitge; Spain) illustrated the contribution of PI3K in blood vessel formation and angiogenesis. She showed that whereas the PI3K $\alpha$ isoform is essential for endothelial cell differentiation, $\mathrm{PI} 3 \mathrm{~K} \beta$ is essential for pericyte-dependent formation of the outer cell layer of the vessels (Okkenhaug et al., 2016). These functions should be considered as a potential advantage (or a side effect) when using PI3K inhibitors for tumor treatment. Mariona also reviewed the consequences of expressing PI $3 \mathrm{~K} \alpha$-activating mutations in different tissues. Expression of a $\mathrm{PI} 3 \mathrm{~K} \alpha$-activating mutation in germ tissues is lethal for the embryo. When the mutation arises in somatic tissue that derives from mesoderm (including blood vessels), it leads to development of an overgrowth syndrome, while the appearance of these mutations in epithelial cells causes cancer.

Another cell response affected by PI3K deregulation is metabolism. Arkaitz Carracedo (CIC bioGUNE, Spain) explained how the metabolic changes detected in cancer are often induced by deregulation of PI3K or TOR, or their regulators. He showed that these changes not only depend on the tumor type, but also on the tumor stage and on the particular set of 'driving mutations' from which the tumor originates. He examined the changes observed in a prostate cancer model and found that the double loss-of-function mutation of PTEN and PGC $\alpha$ (also known as PPARGC1A) increases the metastatic potential of these tumors. Conversely, the increase in PGC $\alpha$ activity correlates with a better prognosis. Activation of the PI3K/mTOR axis is a central node at the foundation of metabolic changes in cancer; as these changes might affect the metastatic potential of tumors and cancer survival, the analysis of the particular metabolic alterations in tumors might be useful in the diagnosis and treatment of neoplastic disease.

Pascale Zimmermann (Department of Human Genetics, Leuven, Belgium \& CRCM, Marseille, France) and Volker Haucke (FMP, Freie Universität Berlin, Germany) discussed the importance of intracellular trafficking in the progression of cancer and the release of extracellular vesicles. Pascale presented her results on the mechanisms of formation of exosomes, structures that mediate communication between neighboring and distant cells. She recommended using the nomenclature 'extracellular vesicles' (EVs) if the origin of a vesicle cannot be confirmed. She described the regulation of these EVs by Src (Imjeti et al., 2017). Volker presented his latest findings on the mechanism by which PI3K-C $2 \beta$ represses the activity of the nutrient sensor mTOR. He also showed exciting data suggesting that PI3K-C2 $\beta$ itself is regulated by an upstream kinase that functions as a novel component of the oncogenic PI3K-mTOR pathway.

Overall, the observations presented in this section show that, besides tumor cell division, the activation of PI3K/mTOR affects tumor metabolism, the formation of blood vessels around the tumor and also intracellular trafficking. These effects should be considered at the time of tumor classification and when making treatment decisions.

\section{Cooperation of PI3K with other 'drivers' and alterations in cancer}

The p53-encoding gene TP53, the most frequently mutated tumor suppressor in human cancer, is degraded in physiological conditions owing to the actions of the associated Mdm2 E3 ubiquitin ligase.
Under stress conditions, p53 migrates to the nucleus and is stabilized. We presented our studies on the process of nuclear p53 stabilization (Richard A. Anderson, University of WisconsinMadison, WI). Following a stress event (such as DNA damage or oxidative stress), p53 associates with the nuclear PI(4)P 5-kinase PIPKI $\alpha$, which supports p53 binding to $\mathrm{PI}(4,5) \mathrm{P} 2$. The binding of PI(4,5)P2 stabilizes p53 by promoting the interaction with small heat-shock proteins (HSP27 and others). Mutant p53 constitutively associates with PIPKI $\alpha$ and $\operatorname{PI}(4,5) \mathrm{P} 2$, and is stabilized by this mechanism. The PI(4,5)P2 that is bound to both wild-type and mutant p53 can be phosphorylated by nuclear IMPK to generate p53-PI(3,4,5)P3, which in turn can be dephosphorylated by nuclear PTEN. The binding of these phosphoinositide-metabolizing enzymes and lipids to p53 controls both wild-type and mutant p53 stability and function.

Continuing with p53 was Lloyd C. Trotman (Cold Spring Harbor, NY) who studies the cooperation of the tumor suppressor genes PTEN and TP53. Whereas p53 is able to trigger PTEN expression (Stambolic et al., 2001), PTEN mutation is known to reduce p53 levels (Freeman et al., 2003), showing that these tumor suppressors co-regulate each other; the double PTEN and TP53 mutation is frequent in cancer, and the phenotype of these tumors should be studied to design therapies. Using a tumor model in which Pten and Tp53 are simultaneously eliminated in the mouse prostate, he showed that loss or pharmacological inhibition of the phosphatase Phlpp2 triggers cell death in PTEN- and p53-deficient tumor cells. Even though Phlpp2 suppresses the action of AKT (Brognard and Newton, 2008; Nowak et al., 2015), it is also a regulator of Myc oncogene stability. The inhibition of Phlpp2 promotes the degradation of Myc, thereby inducing regression of the PTENand p53-mutant tumors.

One of the driving events in both cancer and aging is activation of telomerase. Telomere stability is also regulated by PI3K activity. Maria A. Blasco (Spanish National Cancer Research Centre, Spain) presented her studies on the protection of telomere stability by PI3K. They found that AKT phosphorylates TRF1, a component of the 'shelterin' complex that safeguards telomere integrity (Bejarano et al., 2017) (Fig. 1). The inhibition of PI3K $\alpha$ induces telomere damage and chromosomal instability and should be considered for PI3K-inhibitor planned therapies. The regulation of the phosphorylation of TRF1 must be a critical event for telomeric protection, as not only PI3K/AKT, but also other important cancer kinases, regulate TRF1 phosphorylation.

These observations unmask different levels of cooperation of PI3K/PTEN in cancer; not only do phospholipids seem to stabilize wild-type and mutant p53, but activating mutations in PI3K might favor telomere stability. The oncogenic potential of the double PTEN and p53 mutations could be restricted by the blockage of Phlpp2.

\section{Lessons learned for future cancer treatment}

We presented our data suggesting that not only can gene amplifications and gene mutations act as tumor drivers; in addition, increased expression of PI3K regulators can also promote carcinogenesis (Ana Carrera, Centro Nacional de Biotecnología/CSIC, Spain). This is indeed the case for $p 85 \beta$, a regulatory component of class I PI3K proteins that is expressed at low levels in most normal cells that exhibit preferential expression of p $85 \alpha$ (Fig. 1). p $85 \beta$ levels, however, increase in several tumor types and contribute to accelerate tumor progression and metastasis. Reduction of $\mathrm{p} 85 \beta$ levels is therapeutic in mouse-grown tumors, indicating that the increase of PIK3R2 (the gene encoding $\mathrm{p} 85 \beta$ ) expression can act as a driver event in cancer (Vallejo-Díaz et al., 
2016; Cortés et al., 2012). Detection of PI3K regulators that act as drivers in cancer might help in the stratification of PI3K-active tumors. An increase in $\mathrm{p} 85 \beta$ expression should be considered for the design of new therapies aimed at interfering with PI3K action.

Bart Vanhaesebroeck (UCL Cancer Institute, UK) discussed possible alternatives to the therapy protocols that are currently used with PI3K inhibitors. Given that PI3K $\alpha$-activating mutations mediate a moderate activation of the pathway, he argues that it might make more sense to treat patients with low doses of PI3K inhibitors to prevent the inactivation of the negative-feedback loops or the acquisition of resistance (Semple and Vanhaesebroeck, 2018). He also discussed the potential utility of inhibitors of PI3K isoforms expressed in hematopoietic cells for use in cancer immunotherapy for solid tumors, and how an adaptive immune response most likely also contributes to the clinical efficacy of the PI $3 \mathrm{~K} \delta$ inhibitors in Bcell malignancies.

Along the same line, Klaus Okkenhaug (University of Cambridge, UK) presented an update on the use of inhibitory compounds for $\mathrm{PI} 3 \mathrm{~K} \delta$ (an isoform expressed mainly in the hematopoietic system) for the treatment of B-cell malignancies. Based on his previous description of the contribution of PI $3 \mathrm{~K} \delta$ in the differentiation of regulatory $\mathrm{T}$ cells, which inhibit the effector cytotoxic $\mathrm{T}$ cells, he proposes that $\mathrm{PI} 3 \mathrm{~K} \delta$ inhibition in cancer might render effector $\mathrm{T}$ cells more active against the tumor. PI $3 \mathrm{~K} \delta$ inhibitors could therefore be useful in immunotherapy by reducing regulatory $\mathrm{T}$ cells. However, when tested, they found that PI3K $\delta$ inhibitors did not cooperate with therapies directed to block CTLA4 or PD1. By contrast, PI3K $\delta$ inhibitors synergized with inhibitors for CSF1R (Lim et al., 2018). A possible explanation for this cooperation could be that CSF1 activates macrophages, which in turn facilitate metastasis of surrounding tumor cells.

This session discussed several aspects that might improve the efficacy of PI3K inhibitors for cancer treatment. In addition to scoring PI3K/PTEN mutations, we should consider that the expression of PI3K regulators, such as $\mathrm{p} 85 \beta$, might also make a tumor dependent on PI3K activity. A change in protocols for compound administration (lowering the doses) and the use of selective PI3K inhibitors, as well as inhibitors of the hematopoietic isoforms (PI3K $\gamma$ and PI3K $\delta$ ) for immunotherapy, should also be borne in mind for the future.

Taken together, the data discussed here have enriched our understanding of the mechanisms of tumorigenesis induced by $\mathrm{PI} 3 \mathrm{~K}$, its actions on the organism, and the resistance mechanisms generated upon treatment with PI3K inhibitors. This should help to delineate new strategies for cancer treatments aimed at blocking the action of $\mathrm{PI} 3 \mathrm{~K}$

\section{Acknowledgements}

The meeting has been financed by the Biochemical Journal, The Company of Biologists Limited (www.biologists.com) and one of its journals, Journal of Cell Science. Work in the group of ACC is financed by grants from the Ministerio de Ciencia e Innovación (Spanish Ministry of Science and Innovation; SAF201679195), the Madrid regional government (BMD-3804), the Fundación Ramón Areces and the Fundación Científica Asociación Española Contra el Cáncer (16035CARR).

\section{Competing interests}

The authors declare no competing or financial interests.

\section{References}

Bejarano, L., Schuhmacher, A. J., Méndez, M., Megías, D., Blanco-Aparicio, C. Martínez, S., Pastor, J., Squatrito, M. and Blasco, M. A. (2017). Inhibition of TRF1 telomere protein impairs tumor initiation and progression in glioblastoma mouse models and patient-derived xenografts. Cancer Cell 32, 590-607.e4.

Brognard, J. and Newton, A. C. (2008). PHLiPPing the switch on Akt and protein kinase C signaling. Trends Endocrinol. Metab. 19, 223-230.
Campa, C. C., Margaria, J. P., Derle, A., Del Giudice, M., De Santis, M. C. Gozzelino, L., Copperi, F., Bosia, C. and Hirsch, E. (2018). Rab11 activity and Ptdlns(3)P turnover removes recycling cargo from endosomes. Nat. Chem. Biol. 14, 801-810.

Cortés, I., Sánchez-Ruíz, J., Zuluaga, S., Calvanese, V., Marqués, M., Hernández, C., Rivera, T., Kremer, L., González-García, A. and Carrera, A. C. (2012). p85 phosphoinositide 3-kinase subunit regulates tumor progression. Proc. Natl. Acad. Sci. USA 109, 11318-11323.

Denley, A., Kang, S., Karst, U. and Vogt, P. K. (2007). Oncogenic signaling of class I PI3K isoforms. Oncogene 18, 2561-2574

Franco, I., Gulluni, F., Campa, C. C., Costa, C., Margaria, J. P., Ciraolo, E. Martini, M., Monteyne, D., De Luca, E., Germena, G. et al. (2014). PI3K class II $\alpha$ controls spatially restricted endosomal Ptdlns3P and Rab11 activation to promote primary cilium function. Dev. Cell 28, 647-658.

Franke, T. F., Kaplan, D. R., Cantley, L. C. and Toker, A. (1997). Direct regulation of the Akt proto-oncogene product by phosphatidylinositol-3,4-bisphosphate. Science 275, 665-668.

Freeman, D. J., Li, A. G., Wei, G., Li, H.-H., Kertesz, N., Lesche, R., Whale, A. D., Martinez-Diaz, H., Rozengurt, N., Cardiff, R. D. et al. (2003). PTEN tumor suppressor regulates p53 protein levels and activity through phosphatasedependent and -independent mechanisms. Cancer Cell 3, 117-130.

Gulluni, F., Martini, M., De Santis, M. C., Campa, C. C., Ghigo, A., Margaria, J. P., Ciraolo, E., Franco, I., Ala, U., Annaratone, L. et al. (2017). Mitotic spindle assembly and genomic stability in breast cancer require PI3K-C2 $\alpha$ scaffolding function. Cancer Cell 32, 444-459.e7.

Hill, R., Kalathur, R. K. R., Colaço, L., Brandão, R., Ugurel, S., Futschik, M. and Link, W. (2015). TRIB2 as a biomarker for diagnosis and progression of melanoma. Carcinogenesis 36, 469-477.

Hill, R., Madureira, P. A., Ferreira, B., Baptista, I., Machado, S., Colaço, L., Dos Santos, M., Liu, N., Dopazo, A., Ugurel, S. et al. (2017). TRIB2 confers resistance to anti-cancer therapy by activating the serine/threonine protein kinase AKT. Nat. Commun. 8, 14687

Houslay, D. M., Anderson, K. E., Chessa, T., Kulkarni, S., Fritsch, R. Downward, J., Backer, J. M., Stephens, L. R. and Hawkins, P. T. (2016). Coincident signals from GPCRs and receptor tyrosine kinases are uniquely transduced by PI3K $\beta$ in myeloid cells. Sci. Signal. 9, ra82.

Imjeti, N. S., Menck, K., Egea-Jimenez, A. L., Lecointre, C., Lembo, F., Bouguenina, H., Badache, A., Ghossoub, R., David, G., Roche, S. et al. (2017). Syntenin mediates SRC function in exosomal cell-to-cell communication. Proc. Natl. Acad. Sci. USA 114, 12495-12500.

Jiménez, C., Hernández, C., Pimentel, B. and Carrera, A. C. (2002). The p85 regulatory subunit controls sequential activation of phosphoinositide 3-kinase by Tyr kinases and Ras. J. Biol. Chem. 277, 41556-41562.

Khalil, B. D., Hsueh, C., Cao, Y., Abi Saab, W. F., Wang, Y., Condeelis, J. S. Bresnick, A. R. and Backer, J. M. (2016). GPCR signaling mediates tumor metastasis via PI3K $\beta$. Cancer Res. 76, 2944-2953.

Lim, E. L., Cugliandolo, F. M., Rosner, D. R., Gyori, D., Roychoudhuri, R. and Okkenhaug, K. (2018). Phosphoinositide 3-kinase $\delta$ inhibition promotes antitumor responses but antagonizes checkpoint inhibitors. JCl Insight 3, 120626.

Link, W. (2015). Tribbles breaking bad: TRIB2 suppresses FOXO and acts as an oncogenic protein in melanoma. Biochem. Soc. Trans. 43, 1085-1088.

Liu, P., Cheng, H., Roberts, T. M. and Zhao, J. J. (2009). Targeting the phosphoinositide 3-kinase pathway in cancer. Nat. Rev. Drug Discov. 8, 627-644

Malik, N., Macartney, T., Hornberger, A., Anderson, K. E., Tovell, H., Prescott A. R. and Alessi, D. R. (2018). Mechanism of activation of SGK3 by growth factors via the Class 1 and Class 3 PI3Ks. Biochem. J. 475, 117-135.

Martincorena, I. and Campbell, P. J. (2015). Somatic mutation in cancer and normal cells. Science 349, 1483-1489.

Nowak, D. G., Cho, H., Herzka, T., Watrud, K., DeMarco, D. V., Wang, V. M. Y., Senturk, S., Fellmann, C., Ding, D., Beinortas, T. et al. (2015). MYC drives Pten/Trp53-deficient proliferation and metastasis due to IL6 secretion and AKT suppression via PHLPP2. Cancer Discov. 5, 636-651.

Ohashi, Y., Tremel, S. and Williams, R. L. (2018). VPS34 complexes from a structural perspective. J. Lipid Res. 2018, jlr.R089490.

Okkenhaug, K., Graupera, M. and Vanhaesebroeck, B. (2016). Targeting PI3K in cancer: impact on tumor cells, their protective stroma, angiogenesis, and immunotherapy. Cancer Discov. 6, 1090-1105.

Posor, Y., Eichhorn-Gruenig, M., Puchkov, D., Schöneberg, J., Ullrich, A., Lampe, A., Müller, R., Zarbakhsh, S., Gulluni, F., Hirsch, E. et al. (2013) Spatiotemporal control of endocytosis by phosphatidylinositol-3,4-bisphosphate. Nature 499, 233-237.

Semple, R. K. and Vanhaesebroeck, B. (2018). Lessons for cancer drug treatment from tackling a non-cancerous overgrowth syndrome. Nature 558, 523-525.

Stambolic, V., MacPherson, D., Sas, D., Lin, Y., Snow, B., Jang, Y., Benchimol, S. and Mak, T. W. (2001). Regulation of PTEN transcription by p53. Mol. Cell 8 , 317-325

Vallejo-Díaz, J., Olazabal-Morán, M., Cariaga-Martínez, A. E., Pajares, M. J., Flores, J. M., Pio, R., Montuenga, L. M. and Carrera, A. C. (2016). Targeted depletion of PIK3R2 induces regression of lung squamous cell carcinoma Oncotarget 7, 85063-85078. 